\title{
High-Resolution Frequency Estimation Using Generalized Coprime Sampling
}

\author{
Si Qin, Yimin D. Zhang, and Moeness G. Amin \\ Center for Advanced Communications, Villanova University, Villanova, PA 19085, USA
}

\begin{abstract}
The coprime sampling scheme allows signal frequency estimation through two sub-Nyquist samplers where the down-sampling rates $M$ and $N$ are coprime integers. By considering the difference set of this pair of $O(M+N)$ physical samples, $O(M N)$ consecutive virtual samples can be generated. In this paper, a generalized coprime sampling technique is proposed by using $O(M+p N)$ samples to generate $O(p M N)$ virtual samples, where $p$ is an integer argument. As such, the existing coprime sampling techniques are represented as a special case of a much broader and generalized scheme. The analytical expressions of the number of virtual samples, frequency resolution and the corresponding latency time are derived. The effectiveness of the proposed technique is verified using simulation results.
\end{abstract}

Keywords: Coprime sampling, sparse sampling, super resolution, spectrum estimation

\section{INTRODUCTION}

Spectrum estimation is an important area that finds variety of applications in sensing, surveillance, and communications [1-3]. It is well known that the spectrum of a signal can be uniquely determined if the sampling rate satisfies the Nyquist theorem. However, as the growing demand for higher signal bandwidth, the Nyquist sampling requirement puts enormous demands on sensors, instruments and measurement devices, e.g., the analog to digital converter (ADC).

To solve that problem, frequency determination using sparse sampling has received rapidly increasing interest. In particular, sampling of a signal using multiple samples with sub-Nyquist sampling rates is proposed in $[4,5]$. Among a number of techniques that are available for sparse sampling, coprime sampling is very attractive because it enables a systematical design capability and degree-of-freedom (DOF) analysis [6-9]. Coprime sampling was first used for identifying deterministic sinusoids in noise [6]. Then, the corresponding robust version was proposed in [7] because of the inevitable presence of noise. However, such algorithms require a large number of samplers to identify multiple frequencies. The wide sense stationary (WSS) scenarios were discussed in [8]. Only two mutually coprime samplers are used with sample respective intervals of $M T_{s}$ and $N T_{s}$, where $M$ and $N$ are coprime integers representing down-sampling rates, and $T_{s}$ is the original sampling interval, which is determined by the spectrum bandwidth $B_{s}$. A larger positive semi-definite matrix with all correlation lags from $-M N$ to $M N$ can be obtained, based on the difference set between $2 N$ samples from the former sampler and $M$ samples from the latter sampler. Then the spectrum can be estimated with a resolution proportional to $2 \pi /\left(M N T_{s}\right)$ by directly applying subspace-based technique, e.g., MUSIC [10]. As a result, it allows for identification up to $M N$ frequencies. The multidimensional coprime sensing was then extended from the previous implementations to acquire densely sampled domain in [9]. In this case, the number of detectable frequencies and frequency resolution of such those technique increase as $M$ and $N$ increase. However, it should be noted that the latency time, which is determined by the data updating cycle, also significantly increases.

In this paper, a generalized coprime sampling technique for WSS signals is proposed by using a longer time period used for correlation entry estimation. The generalized coprime concept was initially proposed in the context of array configurations for direction-of-arrival estimation $[11,12]$. In this paper, we extend the result to the time domain. In particular, we introduce an integer factor $p$, such that $p N$ samples in the first sampler, in lieu of $2 N$, are used to estimate correlation entries. As such, the existing coprime sampling technique is represented as a special case of $p=2$. It is shown that the use of a large value of $p$ enables improved frequency

Contact information: yimin@ieee.org. 
resolution and estimation performance. We derive the expressions of frequency resolution and the latency time for quantitative evaluation, comparison, and optimal design, with respect to integer factor $p$. The effectiveness of the proposed technique is verified using simulation results.

Notations: We use lower-case (upper-case) bold characters to denote vectors (matrices). In particular, $\mathbf{I}_{N}$ denotes the $N \times N$ identity matrix. (.) $)^{*}$ implies complex conjugation, whereas $(.)^{T}$ and $(.)^{H}$ respectively denote the transpose and conjugate transpose of a matrix or a vector. $\mathrm{E}($.$) is the statistical expectation operator.$

\section{THEORY AND PROPERTIES FOR COPRIME SAMPLING}

As illustrated in Fig. 1, there are two sets of uniformly spaced samples of the input WSS signal $X(t)$ using two samplers, with sampling intervals $M T_{s}$ and $N T_{s}$, respectively. $T_{s}$ is a unit sampling interval that satisfies the Nyquist theorem. Denote $x[n]$ as the discretized waveforms of $X\left(n T_{s}\right)$. Then, the two sampled stream outputs can be expressed as

$$
\begin{aligned}
& x_{1}\left[n_{1}\right]=x\left[M n_{1}\right]=X\left(M n_{1} T_{s}\right), \\
& x_{2}\left[n_{2}\right]=x\left[N n_{2}\right]=X\left(N n_{2} T_{s}\right) .
\end{aligned}
$$

For non-negative integer $l$, there are no overlapping entries between such two sets other than $x[M N l]$.

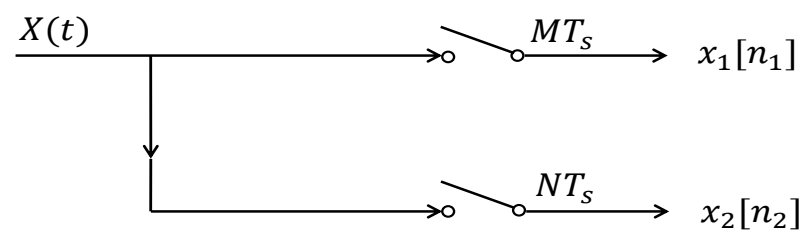

Figure 1: Coprime sampling structure.

The correlation between the two sample sets, i.e., $\mathrm{E}\left[x_{1}\left[n_{1}\right] x_{2}^{*}\left[n_{2}\right]\right]$, yields the $\left(n_{1}, n_{2}\right)$ th entry in the covariance matrix with the lag

$$
k=M n_{1}-N n_{2} .
$$

It has been demonstrated in [8] that, due to the coprimality of $M$ and $N$, we can always find $n_{1}$ and $n_{2}$ in the range $0 \leq n_{1} \leq 2 N-1$ and $0 \leq n_{2} \leq M-1$, for any integer $k$ in the range $0 \leq k \leq M N-1$. The lags will also occupy the range $-M N+1 \leq k \leq 0$ but with some missing entries. The correlation at these missing lags, however, can be obtained from the symmetric positive counterparts through conjugation. As such, we can obtain the correlation entries $R(k)$, for $k=-M N+1, \ldots, M N-1$. In practice, the expectation is approximated by sample average. Since Eqn. (2) can be rewritten as $k=M\left(n_{1}+N l\right)-N\left(n_{2}+M l\right)$ for any integer $l$, the following full-rank Toeplitz correlation matrix can be estimated as

$$
\mathbf{R}_{\text {est }}=\left(\begin{array}{cccc}
R_{\text {est }}(0) & R_{\text {est }}(-1) & \ldots & R_{\text {est }}(-M N+1) \\
R_{\text {est }}(1) & R_{\text {est }}(0) & \ldots & R_{\text {est }}(-M N+2) \\
\vdots & \vdots & \ldots & \vdots \\
R_{\text {est }}(M N-1) & R_{\text {est }}(M N-2) & \ldots & R_{\text {est }}(0)
\end{array}\right)
$$

with the lag $k$ coefficient expressed as

$$
R_{\mathrm{est}}(k)=\left\{\begin{array}{l}
\frac{1}{L} \sum_{l=0}^{L-1} x\left[M\left(n_{1}+N l\right)\right] x^{*}\left[N\left(n_{2}+M l\right)\right], k \in[0, M N-1], \\
R_{\mathrm{est}}^{*}(-k), k \in[-M N+1,0),
\end{array}\right.
$$

where $n_{1} \in[0,2 N-1], n_{2} \in[0, M-1]$, and $L$ is the number of time domain blocks used in averaging. The matrix can then be used to estimate the spectrum using discrete Fourier transform (DFT) or subspace-based 
techniques. It is noted that the spectrum with bandwidth $B_{s}$ can be entirely identified since the covariance matrix $\mathbf{R}_{\text {est }}$ contains all lags with a sampling rate $f_{s}=1 / T_{s}$. In addition, while there are some values out of the range $[-M N, M N]$, only the consecutive lags range in Eqn. (2) can be used to estimate the correlations, since the eigen-decomposition operation will be performed with respect to the toeplitz matrix $\mathbf{R}_{\text {est }}$. Such a technique, which uses $2 N$ samples in $x_{1}[n]$ and $M$ samples in $x_{2}[n]$ to generate correlation entries, is described in [8] and is referred to as conventional coprime sampling in this paper. For better illustrative purpose, the following properties are summarized.

A. The maximum sampling rate of the two samplers is the larger value of $f_{s} / M$ and $f_{s} / N$.

B. This technique can estimate up to $M N$ frequencies in the spectrum, with a frequency resolution proportional to $f_{s} /(M N)$.

C. The latency time $T_{l}$, which is defined as the time to collect samples, is the larger value of sampling time with respect to the two samplers, expressed as

$$
T_{l}=[M(2 N-1)+M N(L-1)] T_{s}=[M N L+M(N-1)] T_{s} .
$$

\section{GENERALIZED COPRIME SAMPLING}

Based on the properties in the previous section, the spectrum can be estimated using the coprime sampling structure with low sub-Nyquist rates. By increasing the values of $M$ and $N$, the number of detectable frequencies increases and the frequency resolution becomes finer. While the sampling may be arbitrarily sparse, it should be noted that the latency time, which is approximately $M N L T_{s}$, increases with both $M$ and $N$. On the other hand, there are less samples in constant data updating cycle, resulting in the deterioration of the spectrum estimation performance. In this section, a generalized coprime sampling technique is proposed to improve the frequency resolution and estimation performance with almost the same latency time.

An integer factor $p$ is introduced to achieve a larger number of lags. Instead of using $2 N$ samples in the first sampler as in the conventional coprime sampling scheme, we use $p N$ samples in the first sampler to estimate correlation matrix, whereas the same $M$ samples are used in the second sampler. In this case, the resulting lags fall into the following set,

$$
\mathbb{K}=\left\{k \mid k=M n_{1}-N n_{2}, 0 \leq n_{1} \leq p N-1,0 \leq n_{2} \leq M-1\right\} .
$$

For different factor $p$, we obtain different set $\mathbb{K}$. An example is shown in Fig. 2. It is observed from this figure that the number of positive lags increases as $p$ increases from 2 to 4 . The following proposition about set $\mathbb{K}$ is useful to understand the property of the resulting lag positions.

Proposition1 : The set $\mathbb{K}$ contains all the consecutive integers in the range $0 \leq k \leq(p-1) M N+N-1$.

Proof: Given any integer $k$ satisfying

$$
0 \leq k \leq(p-1) M N+N-1,
$$

we need to prove that there exist integers $n_{1} \in[0, p N-1]$ and $n_{2} \in[0, M-1]$ such that $k=M n_{1}-N n_{2}$ holds. The requirement $n_{2} \in[0, M-1]$ is equivalent to

$$
0 \leq N n_{2} \leq M N-N
$$

Because $M n_{1}=k+N n_{2}$, we obtain the following relationship by combining (7) and (8),

$$
0 \leq M n_{1} \leq p M N-1<p M N .
$$

This result can be equivalently expressed as $0 \leq n_{1}<p N$. Because $n_{1}$ is an integer, this requirement is equivalent to

$$
0 \leq n_{1} \leq p N-1,
$$




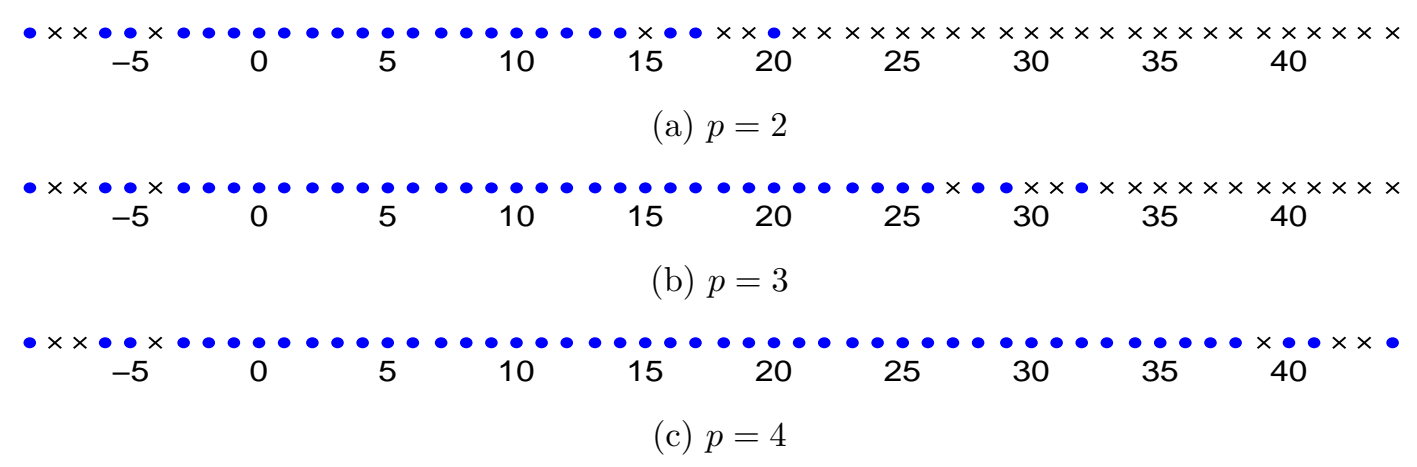

Figure 2: The set $\mathbb{K}$ with different values of $p(M=4$ and $N=3$. •: Valid lag positions; $\times$ : missing lag positions).

which is satisfied in the underlying coprime array configuration.

As a result of proposition 1 , the correlation matrix $\tilde{\mathbf{R}}_{\text {est }}$ can be systematically obtained with the entries

$$
\tilde{R}_{\mathrm{est}}(k)=\left\{\begin{array}{l}
\frac{1}{L} \sum_{l=0}^{L-1} x\left[M\left(n_{1}+N l\right)\right] x^{*}\left[N\left(n_{2}+M l\right)\right], k \in[0,(p-1) M N+N-1], \\
\tilde{R}_{\mathrm{est}}^{*}(-k), k \in[-(p-1) M N-N+1,0),
\end{array}\right.
$$

where $n_{1} \in[0, p N-1]$ and $n_{2} \in[0, M-1]$. It becomes conventional coprime sampling when $p=2$. Thus, the conventional coprime sampling case can be considered as a special case of the generalized coprime sampling. As a comparison, the corresponding properties of generalized coprime sampling are summarized below:

A. The maximum sampling rate remains the larger value of $f_{s} / M$ and $f_{s} / N$.

B. It can estimate up to $(p-1) M N+N-1$ frequencies in the spectrum, which is increased by a factor of $p-1$.

C. The frequency resolution is approximately proportional to $f_{s} /[(p-1) M N]$, which is an improvement by a factor of $p-1$.

D. The latency time $T_{l}$ is

$$
T_{l}=[M(p N-1)+M N(L-1)] T_{s}=(M N L-M) T_{s}+(p-1) M N T_{s},
$$

which is approximately the same as the conventional coprime sampling, particularly for a large number of time blocks $L$.

Therefore, when $L$ is large, a high value of $p$ in the generalized coprime sampling scheme leads to an improved estimation performance without increasing the values of $M$ and $N$ as in conventional coprime sampling. The maximum number of $p$ is bounded by the minimum updating data rate of the system determined by $M, N$, $L$ and $T_{s}$. Since we obtain the estimated correlation based on the received data samples as in Eqn. (11), the number of time blocks $L$ takes a large value to reduce the noise perturbation. In that case, the second term in Eqn. (12) is negligible when $p \ll L$, compared to the corresponding first term, and $T_{l}$ keeps nearly constant as $p$ increases.

\section{SIMULATION RESULTS}

Simulation results are presented to demonstrate the effectiveness of the proposed technique for different values of $p$. Cases of $p=2$ (or conventional coprime sampling), $p=5$ and $p=30$ are considered. In all simulations, we assume $Q$ sinusoidal signals with frequencies $f_{q}$, for $q=1, \ldots, Q$, which are distributed in $B_{s}=[0,300] \mathrm{MHz}$ 
with the original sampling rate $f_{s}=600 \mathrm{MHz}$. The noise power is assumed to be constant with this frequency band. $M=4$ and $N=3$ are considered, i.e., the sub-Nyquist sampling rates for the two samples are respectively $f_{s} / M=150 \mathrm{MHz}$ and $f_{s} / N=200 \mathrm{MHz}$. In addition, $L=800$ are used to estimate the correlations $\tilde{R}_{\text {est }}(k)$ with $k \in[-(p-1) M N-N+1,(p-1) M N+N-1]$, i.e., $[-14,14],[-50,50]$ and $[-350,350]$, respectively. We use the MUSIC algorithm in time domain to perform the frequency identification.

\subsection{The number of detectable frequencies}

We first compare the number of detectable frequencies. $Q=61$ frequencies, which are uniformly distributed between $5 \mathrm{MHz}$ and $298 \mathrm{MHz}$, are assumed. Note that, only the $p=30$ case has a sufficient number of DOFs to identify all frequencies. This is verified in Fig. 3 in which only the case of $p=30$ in Fig. 3(c) resolve all 61 frequencies, whereas not all frequencies are correctly identified for the cases of $p=2$ and $p=5$ in Figs. 3(a) and (b).

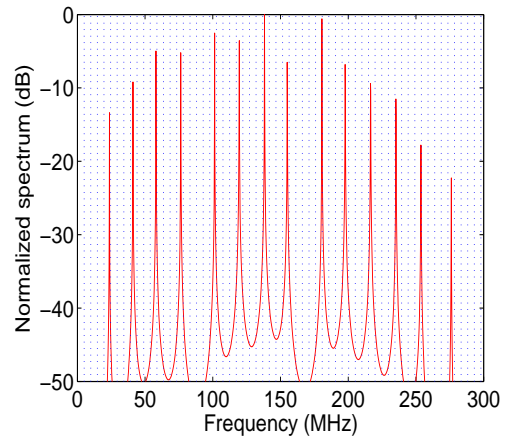

(a)

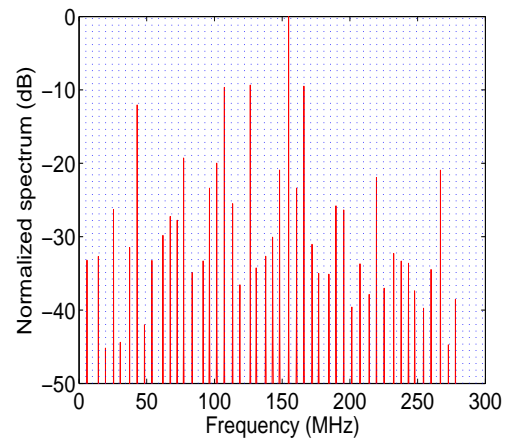

(b)

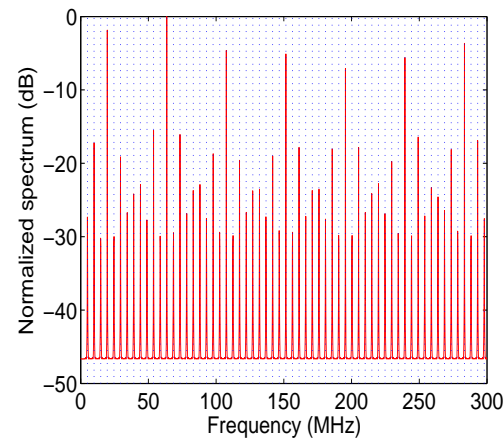

(c)

Figure 3: Estimated spectra for different values of $p(Q=61$ and Input $\mathrm{SNR}=10 \mathrm{~dB})$. (a) $p=2$. (b) $p=5$. (c). $p=30$.

\subsection{Frequency resolution}

In Fig. $4, Q=4$ frequencies, which are located at $[45,45.75,91.5,96] \mathrm{MHz}$, are used to examine the frequency resolution. The result show that, the $p=30$ scenario depicted in Fig. 4(c) can identify all 4 frequencies. However, the case of $p=5$ can only resolve the pair with frequencies $91.5 \mathrm{MHz}$ and $96 \mathrm{MHz}$ but fail to separate the closer pair with frequencies $45 \mathrm{MHz}$ and $45.75 \mathrm{MHz}$, as shown in Fig. 4(b). Both pairs of frequencies are unresolvable when $p=2$, as depicted in Fig. 4(a). Thus, it is evident that the resolution becomes finer as $p$ increases, due to the increased dimension of $\tilde{\mathbf{R}}_{\text {est }}$.

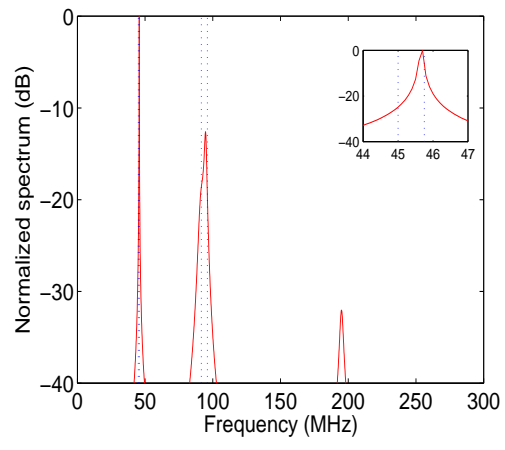

(a)

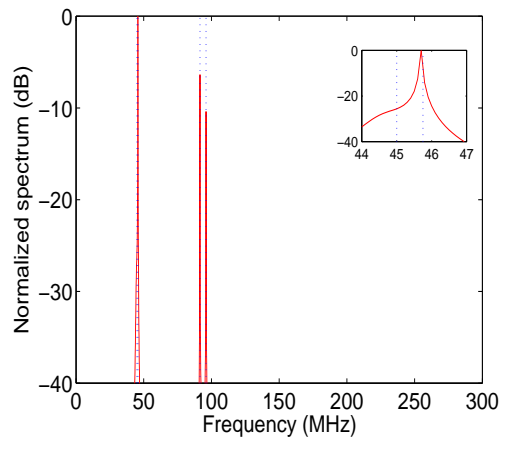

(b)

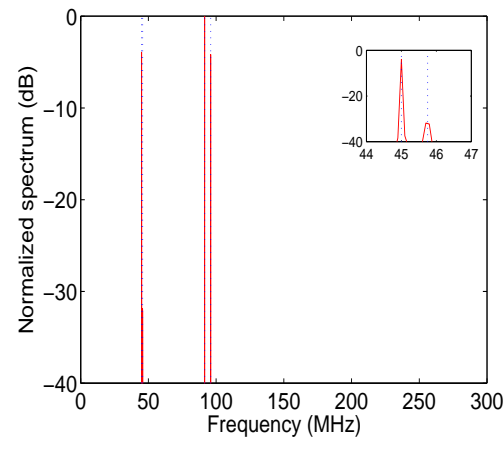

(c)

Figure 4: Estimated spectra for different values of $p(Q=4$ and Input $\mathrm{SNR}=10 \mathrm{~dB})$. (a) $p=2$. (b) $p=5$. (c). $p=30$. 


\subsection{Identification performance}

$Q=4$ frequencies are then changed to be uniformly distributed between $45 \mathrm{MHz}$ and $96 \mathrm{MHz}$, so that their frequency separations satisfy the requirement of the resolution for all cases. We use Monte Carlo simulations to evaluate the average root mean square error (RMSE) of the estimated sinusoidal frequencies, expressed as

$$
\mathrm{RMSE}=\sqrt{\frac{1}{I Q} \sum_{i=1}^{I} \sum_{q=1}^{Q}\left(\hat{f}_{q}(i)-f_{q}\right)^{2}}
$$

where $\hat{f}_{q}(i)$ is the estimate of $f_{q}$ from the $i$ th Monte Carlo trial, $i=1, \ldots, I$. We use $I=5000$ independent trials in the simulations. Fig. 5 compares the RMSE performance as a function of the input SNR. It is evident that the RMSE is improved with the increase of the input SNR. In addition, the performance is improved as the value of $p$ increases.

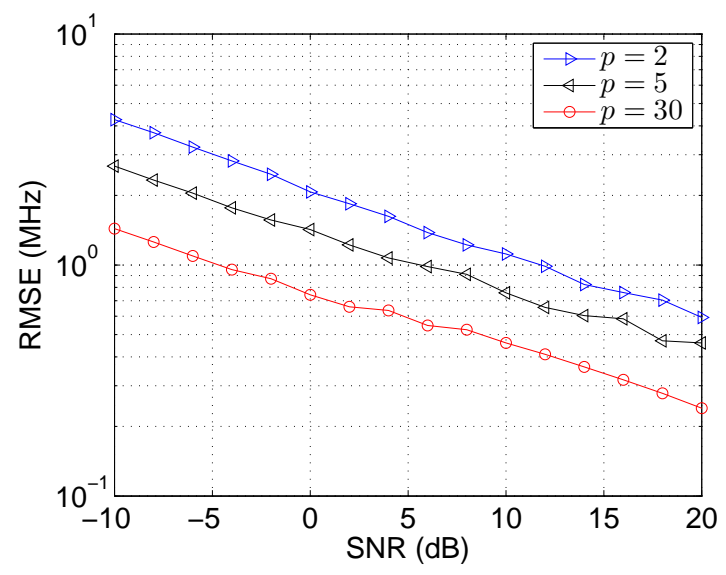

Figure 5: The latency time with respect to the number of time blocks $L$.

\subsection{Latency time}

Finally, we show the latency time, with respect to the number of time blocks $L$, in Fig. 6 based on Eqn. (12). For different values of $p$, it is noted that the latency time slightly increases with $p$, whereas the difference becomes negligible as $L$ increases.

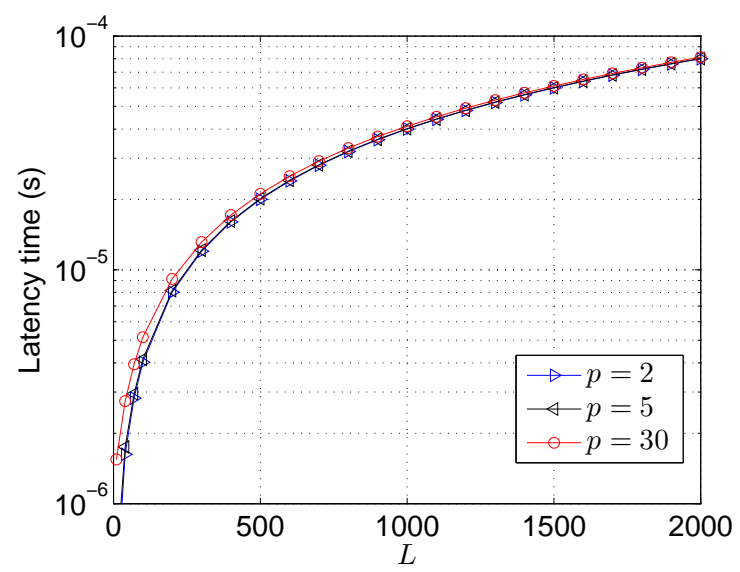

Figure 6: The latency time with respect to the number of time blocks $L$. 


\section{CONCLUSION}

We proposed a generalized coprime sampling technique to estimate the spectra of signals with a coprime pair of sub-Nyquist rate. The proposed approach achieves better frequency resolution and identification performance in a similar latency time. An analytical expression of the number of contiguous lags was derived. The effectiveness of the proposed technique was verified using simulation results.

\section{ACKNOWLEDGMENTS}

This work was supported in part by Office of Naval Research (ONR) under Grant No. N00014-13-1-0061.

\section{REFERENCES}

[1] M. Skolnik, Radar Handbook, Third Edition. New York, NY: McGraw-Hill, 2008.

[2] D. N. C. Tse and P. Viswanath, Fundamentals of Wireless Communications. Cambridge, U.K.: Cambrige Univ. Press, 2005.

[3] Y. Bar-Shalom, X. R. Li, and T. Kirubarajan, Estimation with Applications to Tracking and Navigation: Theory, Algorithms, and Software. New York, NY: Wiley, 2001.

[4] M. D. Zoltowski and C. P. Mathews, "Real-time frequency and 2-D angle estimation with sub-Nyquist spatio-temporal sampling," IEEE Trans. Signal Process., vol. 42, no. 10, pp. 2781-2794, Oct. 1994.

[5] P. E. Pace, R. E. Leino, and D. Styer, "Use of the symmetrical number system in resolving single-frequency undersampled aliases," IEEE Trans. Signal Process., vol. 45, no. 5, pp. 1153-1160, May 1997.

[6] X.-G. Xia, "On estimation of multiple frequencies in undersampled complex value waveforms," IEEE Trans. Signal Process., vol. 47, no. 12, pp. 3417-3419, Dec. 1999.

[7] X.-G. Xia and K. Liu, "A generalized Chinese remainder theorem for residue sets with errors and its application in frequency determination from multiple sensors with low sampling rates," IEEE Signal Process. Lett., vol. 12, no. 11, pp. 768-771, Nov. 2005.

[8] P. P. Vaidyanathan and P. Pal, "Sparse sensing with co-prime samplers and arrays," IEEE Trans. Signal Process., vol. 59, no. 2, pp. 573-586, Feb. 2011.

[9] P. P. Vaidyanathan and P. Pal, "Theory of sparse coprime sensing in multiple dimensions," IEEE Trans. Signal Process., vol. 59, no. 8, pp. 3592-3608, Aug. 2011.

[10] R. O. Schmidt, "Multiple emitter location and signal parameter estimation," IEEE Trans. Antennas Propagat., vol. 34, no. 3, pp. 276-280, March 1986.

[11] S. Qin, Y. D. Zhang, and M. G. Amin, "Generalized coprime array configurations," in Proc. IEEE Sensor Array and Multichannel Signal Processing Workshop, A Coruna, Spain, pp. 529-532, June 2014.

[12] S. Qin, Y. D. Zhang, and M. G. Amin, "Generalized coprime array configurations for direction-of-arrival estimation," IEEE Trans. Signal Process., vol. 63, no. 6, pp. 1377-1390, March 2015. 